\title{
DESINGULARIZATION OF SINGULAR HYPERKÄHLER VARIETIES I
}

\begin{abstract}
Misha Verbitsky
Abstract. Let $M$ be a singular hyperkähler variety, obtained as a moduli space of stable holomorphic bundles on a compact hyperkähler manifold (alg-geom/9307008). Consider $M$ as a complex variety in one of the complex structures induced by the hyperkähler structure. We show that normalization of $M$ is smooth, hyperkähler and does not depend on the choice of induced complex structure.
\end{abstract}

\section{Introduction}

The structure of this paper is as follows.

- In the first section, we give a compendium of definitions and results from hyperkähler geometry, all known from literature.

- Section 2 deals with the real analytic varieties underlying complex varieties. We define almost complex structures on a real analytic variety. This notion is used in order to define hypercomplex varieties. We show that a hyperkähler manifold is always hypercomplex.

- In Section 3, we give a definition of a singular hyperkähler variety, following [V-bun] and [V3]. We cite basic properties and list the examples of such manifolds.

- In Section 4, we define locally homogeneous singularities. A space with locally homogeneous singularities (SLHS) is an analytic space $X$ such that for all $x \in X$, the $x$-completion of a local $\operatorname{ring} \mathcal{O}_{x} X$ is isomorphic to an $x$-completion of associated graded ring $\left(\mathcal{O}_{x} X\right)_{g r}$. We show that a complex variety is SLHS if and only if the underlying real analytic variety is SLHS. This allows us to define invariantly the notion of a hyperkähler SLHS. The natural examples of hyperkähler SLHS include the moduli spaces of stable holomorphic bundles, considered in [V-bun]. ${ }^{1}$ We conjecture that every hyperkähler variety is a space with locally homogeneous singularities.

- In Section 5, we study the tangent cone of a singular hyperkähler manifold $M$ in the point $x \in M$. We show that its reduction, which is a

Received November 11, 1996.

Supported by NSF grant 9304580 .

${ }^{1}$ In [V-bun], we proved that the moduli of stable bundles over a compact hyperkähler manifold is a hyperkähler variety, if we assume certain numerical restrictions on the bundle's Chern classes. The stable bundles satisfying these restrictions are called hyperholomorphic. 
closed subvariety of $T_{x} M$, is a union of linear subspaces $L_{i} \subset T_{x} M$. These subspaces are invariant under the natural quaternion action in $T_{x} M$. This implies that a normalization of $(M, I)$ is smooth. Here, as usually, $(M, I)$ denotes $M$ considered as a complex variety, with $I$ a complex structure induced by the singular hyperkähler structure on $M$.

- In Section 6, we formulate and prove the desingularization theorem for hyperkähler varieties with locally homogeneous singularities. For each such variety $M$ we construct a finite surjective morphism $\widetilde{M} \stackrel{n}{\longrightarrow} M$ of hyperkähler varieties, such that $\widetilde{M}$ is smooth and $n$ is an isomorphism outside of singularities of $M$. The $\widetilde{M}$ is obtained as a normalization of $M$; thus, our construction is canonical and functorial.

\section{Hyperkähler manifolds}

1.1. Definitions. This subsection contains a compression of the basic definitions from hyperkähler geometry, found, for instance, in [Bes] or in [Beau].

Definition 1.1. ([Bes]) A hyperkähler manifold is a Riemannian manifold $M$ endowed with three complex structures $I, J$ and $K$, such that the following holds.

(i): The metric on $M$ is Kähler with respect to these complex structures and

(ii): $I, J$ and $K$, considered as endomorphisms of a real tangent bundle, satisfy the relation $I \circ J=-J \circ I=K$.

The notion of a hyperkähler manifold was introduced by E. Calabi ([C]).

Clearly, hyperkähler manifold has the natural action of quaternion algebra $\mathbb{H}$ in its real tangent bundle $T M$. Therefore its complex dimension is even. For each quaternion $L \in \mathbb{H}, L^{2}=-1$, the corresponding automorphism of $T M$ is an almost complex structure. It is easy to check that this almost complex structure is integrable ([Bes]).

Definition 1.2. Let $M$ be a hyperkähler manifold, $L$ a quaternion satisfying $L^{2}=-1$. The corresponding complex structure on $M$ is called an induced complex structure. The $M$ considered as a complex manifold is denoted by $(M, L)$.

Let $M$ be a hyperkähler manifold. We identify the group $S U(2)$ with the group of unitary quaternions. This gives a canonical action of $S U(2)$ on the tangent bundle, and all its tensor powers. In particular, we obtain a natural action of $S U(2)$ on the bundle of differential forms.

Lemma 1.3. The action of $S U(2)$ on differential forms commutes with the Laplacian.

Proof. This is Proposition 1.1 of [V-bun].

Thus, for compact $M$, we may speak of the natural action of $S U(2)$ in cohomology. 
1.2. Trianalytic subvarieties in compact hyperkähler manifolds. In this subsection, we give a definition and a few basic properties of trianalytic subvarieties of hyperkähler manifolds. We follow [V2].

Let $M$ be a compact hyperkähler manifold, $\operatorname{dim}_{\mathbb{R}} M=2 m$.

Definition 1.4. Let $N \subset M$ be a closed subset of $M$. Then $N$ is called trianalytic if $N$ is a complex analytic subset of $(M, L)$ for any induced complex structure $L$.

Let $I$ be an induced complex structure on $M$, and $N \subset(M, I)$ be a closed analytic subvariety of $(M, I), \operatorname{dim}_{\mathbb{C}} N=n$. Denote by $[N] \in H_{2 n}(M)$ the homology class represented by $N$. Let $\langle N\rangle \in H^{2 m-2 n}(M)$ denote the Poincare dual cohomology class. Recall that the hyperkähler structure induces the action of the group $S U(2)$ on the space $H^{2 m-2 n}(M)$.

Theorem 1.5. Assume that $\langle N\rangle \in H^{2 m-2 n}(M)$ is invariant with respect to the action of $S U(2)$ on $H^{2 m-2 n}(M)$. Then $N$ is trianalytic.

Proof. This is Theorem 4.1 of [V2].

Remark 1.6. Trianalytic subvarieties have an action of quaternion algebra in the tangent bundle. In particular, the real dimension of such subvarieties is divisible by 4 .

\subsection{Totally geodesic submanifolds.}

Proposition 1.7. Let $X \stackrel{\varphi}{\hookrightarrow} M$ be an embedding of Riemannian manifolds (not necessarily compact) compatible with the Riemannian structure. Then the following conditions are equivalent.

(i): Every geodesic line in $X$ is geodesic in $M$.

(ii): Consider the Levi-Civita connection $\nabla$ on $T M$, and restriction of $\nabla$ to $\left.T M\right|_{X}$. Consider the orthogonal decomposition

$$
\left.T M\right|_{X}=T X \oplus T X^{\perp} .
$$

Then, this decomposition is preserved by the connection $\nabla$.

Proof. Well known; see, for instance, [Bes].

Proposition 1.8. Let $X \subset M$ be a trianalytic submanifold of a hyperkähler manifold $M$, where $M$ is not necessarily compact. Then $X$ is totally geodesic.

Proof: This is [V3], Corollary 5.4.

\section{Real analytic varieties}

For the reference and results about real analytic varieties and spaces, see [GMT].

Let $X$ be a complex analytic variety. The "real analytic space underlying $X$ " (denoted by $X_{\mathbb{R}}$ ) is the following object. By definition, $X_{\mathbb{R}}$ is a ringed space 
with the same topology as $X$, but with a different structure sheaf, denoted by $\mathcal{O}_{X_{\mathbb{R}}}$. Let $i: U \hookrightarrow B^{n}$ be a closed embedding of an open subset $U \subset X$ to an open ball $B^{n} \subset \mathbb{C}^{n}$, and $I$ be an ideal defining $i(U)$. Then $\left.\mathcal{O}_{X_{\mathbb{R}}}\right|_{U}$ is a quotient sheaf $\mathcal{O}_{B_{\mathbb{R}}^{n}} / \operatorname{Re}(I)$ of the sheaf of real analytic functions on $B^{n}$ by the ideal $\operatorname{Re}(I)$ generated by the real parts of the functions $f \in I$.

Note that the real analytic space underlying $X$ needs not be reduced.

Consider the sheaf $\mathcal{O}_{X}$ of holomorphic functions on $X$ as a subsheaf of the sheaf $C(X, \mathbb{C})$ of continous $\mathbb{C}$-valued functions on $X$. The sheaf $C(X, \mathbb{C})$ has a natural authomorphism $f \longrightarrow \bar{f}$, where $\bar{f}$ is complex conjugation. By definition, the section $f$ of $C(X, \mathbb{C})$ is called antiholomorphic if $\bar{f}$ is holomorphic. Let $\mathcal{O}_{X}$ be the sheaf of holomorphic functions, and $\overline{\mathcal{O}}_{X}$ be the sheaf of antiholomorphic functions on $X$. Let $\mathcal{O}_{X} \otimes_{\mathbb{C}} \overline{\mathcal{O}}_{X} \stackrel{i}{\longrightarrow} \mathcal{O}_{X_{\mathbb{R}}} \otimes \mathbb{C}$ be the natural multiplication map.

Claim 2.1. The sheaf homomorphism $i: \mathcal{O}_{X} \otimes_{\mathbb{C}} \overline{\mathcal{O}}_{X} \longrightarrow \mathcal{O}_{X_{\mathbb{R}}} \otimes \mathbb{C}$ is injective. For each point $x \in X, i$ induces an isomorphism on $x$-completions of $\mathcal{O}_{X} \otimes_{\mathbb{C}} \overline{\mathcal{O}}_{X}$ and $\mathcal{O}_{X_{\mathbb{R}}} \otimes \mathbb{C}$.

Proof. Clear from the definition.

Let $\Omega^{1}\left(\mathcal{O}_{X_{\mathbb{R}}}\right), \Omega^{1}\left(\mathcal{O}_{X} \otimes_{\mathbb{C}} \overline{\mathcal{O}}_{X}\right), \Omega^{1}\left(\mathcal{O}_{X_{\mathbb{R}}} \otimes \mathbb{C}\right)$ be the sheaves of continuous differentials associated with the corresponding ring sheaves. There are natural sheaf maps

$$
\Omega^{1}\left(\mathcal{O}_{X_{\mathbb{R}}}\right) \otimes \mathbb{C} \longrightarrow \Omega^{1}\left(\mathcal{O}_{X_{\mathbb{R}}} \otimes \mathbb{C}\right)
$$

and

$$
\Omega^{1}\left(\mathcal{O}_{X_{\mathbb{R}}} \otimes \mathbb{C}\right) \longrightarrow \Omega^{1}\left(\mathcal{O}_{X} \otimes_{\mathbb{C}} \overline{\mathcal{O}}_{X}\right)
$$

correspoding to the monomorphisms

$$
\mathcal{O}_{X_{\mathbb{R}}} \hookrightarrow \mathcal{O}_{X_{\mathbb{R}}} \otimes \mathbb{C}, \quad \mathcal{O}_{X} \otimes_{\mathbb{C}} \overline{\mathcal{O}}_{X} \hookrightarrow \mathcal{O}_{X_{\mathbb{R}}} \otimes \mathbb{C}
$$

Claim 2.2. The map (2.1) is an isomorphism. Tensoring both sides of (2.2) by $\mathcal{O}_{X_{\mathbb{R}}} \otimes \mathbb{C}$ produces an isomorphism

$$
\Omega^{1}\left(\mathcal{O}_{X} \otimes_{\mathbb{C}} \overline{\mathcal{O}}_{X}\right) \bigotimes_{\mathcal{O}_{X} \otimes_{\mathbb{C}} \overline{\mathcal{O}}_{X}}\left(\mathcal{O}_{X_{\mathbb{R}}} \otimes \mathbb{C}\right)=\Omega^{1}\left(\mathcal{O}_{X_{\mathbb{R}}} \otimes \mathbb{C}\right)
$$

Proof. Clear.

According to the general results about differentials (see, for example, $[\mathrm{H}]$, Chapter II, Ex. 8.3), the sheaf $\Omega^{1}\left(\mathcal{O}_{X} \otimes_{\mathbb{C}} \overline{\mathcal{O}}_{X}\right)$ admits a canonical decomposition:

$$
\Omega^{1}\left(\mathcal{O}_{X} \otimes_{\mathbb{C}} \overline{\mathcal{O}}_{X}\right)=\Omega^{1}\left(\mathcal{O}_{X}\right) \otimes_{\mathbb{C}} \overline{\mathcal{O}}_{X} \oplus \mathcal{O}_{X} \otimes_{\mathbb{C}} \Omega^{1}\left(\overline{\mathcal{O}}_{X}\right) .
$$


Let $\widetilde{I}$ be an endomorphism of $\Omega^{1}\left(\mathcal{O}_{X} \otimes_{\mathbb{C}} \overline{\mathcal{O}}_{X}\right)$ which acts as a multiplication by $\sqrt{-1}$ on

$$
\Omega^{1}\left(\mathcal{O}_{X}\right) \otimes_{\mathbb{C}} \overline{\mathcal{O}}_{X} \subset \Omega^{1}\left(\mathcal{O}_{X} \otimes_{\mathbb{C}} \overline{\mathcal{O}}_{X}\right)
$$

and as a multiplication by $-\sqrt{-1}$ on

$$
\mathcal{O}_{X} \otimes_{\mathbb{C}} \Omega^{1}\left(\overline{\mathcal{O}}_{X}\right) \subset \Omega^{1}\left(\mathcal{O}_{X} \otimes_{\mathbb{C}} \overline{\mathcal{O}}_{X}\right) .
$$

Let $\underline{I}$ be the corresponding $\mathcal{O}_{X_{\mathbb{R}}} \otimes \mathbb{C}$-linear endomorphism of

$$
\Omega^{1}\left(\mathcal{O}_{X_{\mathbb{R}}}\right) \otimes \mathbb{C}=\Omega^{1}\left(\mathcal{O}_{X} \otimes_{\mathbb{C}} \overline{\mathcal{O}}_{X}\right) \otimes_{\mathcal{O}_{X} \otimes_{\mathbb{C}} \overline{\mathcal{O}}_{X}}\left(\mathcal{O}_{X_{\mathbb{R}}} \otimes \mathbb{C}\right)
$$

As easy check ensures that $\underline{I}$ is real, that is, comes from the $\mathcal{O}_{X_{\mathbb{R}}}$-linear endomorphism of $\Omega^{1}\left(\mathcal{O}_{X_{\mathbb{R}}}\right)$. Denote this $\mathcal{O}_{X_{\mathbb{R}}}$-linear endomorphism by

$$
I: \Omega^{1}\left(\mathcal{O}_{X_{\mathbb{R}}}\right) \longrightarrow \Omega^{1}\left(\mathcal{O}_{X_{\mathbb{R}}}\right),
$$

$I^{2}=-1$. The endomorphism $I$ is called a complex structure operator. In the case when $X$ is smooth, $I$ coinsides with the usual complex structure operator on the cotangent space.

Definition 2.3. Let $X, Y$ be complex analytic varieties, and

$$
f: X_{\mathbb{R}} \longrightarrow Y_{\mathbb{R}}
$$

be a morphism of underlying real analytic spaces. Let $f^{*} \Omega_{Y_{\mathbb{R}}}^{1} \stackrel{P}{\longrightarrow} \Omega_{X_{\mathbb{R}}}^{1}$ be the natural map of sheaves of differentials associated with $f$. Let

$$
I_{X}: \Omega_{X_{\mathbb{R}}}^{1} \longrightarrow \Omega_{X_{\mathbb{R}}}^{1}, \quad I_{Y}: \Omega_{Y_{\mathbb{R}}}^{1} \longrightarrow \Omega_{Y_{\mathbb{R}}}^{1}
$$

be the complex structure operators, and

$$
f^{*} I_{Y}: f^{*} \Omega_{Y_{\mathbb{R}}}^{1} \longrightarrow f^{*} \Omega_{Y_{\mathbb{R}}}^{1}
$$

be $\mathcal{O}_{X_{\mathbb{R}}}$-linear automorphism of $f^{*} \Omega_{Y_{\mathbb{R}}}^{1}$ defined as a pullback of $I_{Y}$. We say that $f$ commutes with the complex structure if

$$
P \circ f^{*} I_{Y}=I_{X} \circ P .
$$

Theorem 2.4. Let $X, Y$ be complex analytic varieties, and

$$
f_{\mathbb{R}}: X_{\mathbb{R}} \longrightarrow Y_{\mathbb{R}}
$$

be a morphism of underlying real analytic spaces, which commutes with the complex structure. Then there exist a morphism $f: X \longrightarrow Y$ of complex analytic varieties, such that $f_{\mathbb{R}}$ is its underlying morphism.

Proof. By Corollary 9.4, [V3], the map $f$, defined on the sets of points of $X$ and $Y$, is meromorphic; to prove Theorem 2.4, we need to show it is holomorphic. Let $\Gamma \subset X \times Y$ be the graph of $f$. Since $f$ is meromorphic, $\Gamma$ is a complex subvariety of $X \times Y$. It will suffice to show that the natural projections $\pi_{1}: \Gamma \longrightarrow X$, 
$\pi_{2}: \Gamma \longrightarrow Y$ are isomorphisms. By [V3], Lemma 9.12, the morphisms $\pi_{i}$ are flat. Since $f_{\mathbb{R}}$ induces isomorphism of Zariski tangent spaces, same is true of $\pi_{i}$. Thus, $\pi_{i}$ are unramified. Therefore, the maps $\pi_{i}$ are etale. Since they are one-to-one on points, $\pi_{i}$ etale implies $\pi_{i}$ is an isomorphism.

Definition 2.5. For a topological space $X$, denote by $C(X, \mathbb{R})$ the sheaf of continous $\mathbb{R}$-values functions on $X$. For $X$ a real analytic space, consider the evaluation map ev : $\mathcal{O}_{X} \longrightarrow C(X, \mathbb{R})$. The kernel $I$ of this map is an ideal sheaf in $\mathcal{O}_{X}$. Consider the ringed space with the same topology as $X$ and with structure sheaf $\mathcal{O}_{X} / I$. This object is called the reduction of $X$, denoted by $X_{r}$. A real analytic space which coinsides with its reduction is called a real analytic variety.

Consider the reduction morphism $X^{r} \stackrel{r}{\hookrightarrow} X$ It is easy to define the functor $r^{*}: S h(X) \longrightarrow S h\left(X^{r}\right)$ of sheaves of $\mathcal{O}_{X}$-modules. Clearly, $\Omega^{1} X^{r}=r^{*} \Omega^{1} X$. Thus, the almost complex structure $I$, if given on $X$, automatically carries over to $X^{r}$. We obtain that a reduction of an almost complex space is an almost complex variety.

Definition 2.6. Let $M$ be a real analytic space, and

$$
I: \Omega^{1}\left(\mathcal{O}_{M}\right) \longrightarrow \Omega^{1}\left(\mathcal{O}_{M}\right)
$$

be an endomorphism satisfying $I^{2}=-1$. Then $I$ is called an almost complex structure on $M$. If there exist a structure $\mathfrak{C}$ of complex variety on $M$ such that $I$ appears as the complex structure operator associated with $\mathfrak{C}$, we say that $I$ is integrable. Theorem 2.4 implies that this complex structure is unique if it exists.

For a real analytic variety $M^{r}$, and an automorphism $I: \Omega^{1}\left(\mathcal{O}_{M^{r}}\right) \longrightarrow$ $\Omega^{1}\left(\mathcal{O}_{M^{r}}\right)$, we say that $I$ is integrable if $M^{r}$ appears as a reduction of some real analytic space with an integrable complex structure.

Definition 2.7. (Hypercomplex variety) Let $M$ be a real analytic variety equipped with almost complex structures $I, J$ and $K$, such that $I \circ J=-J \circ I=$ $K$. Assume that for all $a, b, c \in \mathbb{R}$, such that $a^{2}+b^{2}+c^{2}=1$, the almost complex structure $a I+b J+c K$ is integrable. Then $M$ is called a hypercomplex variety.

Claim 2.8. Let $M$ be a hyperkähler manifold. Then $M$ is hypercomplex.

Proof. Let $I, J$ be induced complex structures. We need to identify $(M, I)_{\mathbb{R}}$ and $(M, J)_{\mathbb{R}}$ in a natural way. These varieties are canonically identified as $C^{\infty}$ manifolds; we need only to show that this identification is real analytic. This is [V3], Proposition 6.5.

The following proposition will be used further on in this paper.

Proposition 2.9. Let $M$ be a complex variety, $x \in X$ a point, and $Z_{x} M \subset$ $T_{x} M$ be the reduction of the Zariski tangent cone to $M$ in $x$, considered as a closed subvariety of the Zariski tangent space $T_{x} M$. Let $Z_{x} M_{\mathbb{R}} \subset T_{x} M_{\mathbb{R}}$ be the Zariski tangent cone for the underlying real analytic space $M_{\mathbb{R}}$. Then $\left(Z_{x} M\right)_{\mathbb{R}} \subset$ $\left(T_{x} M\right)_{\mathbb{R}}=T_{x} M_{\mathbb{R}}$ coinsides with $Z_{x} M_{\mathbb{R}}$. 
Proof. For each $v \in T_{x} M$, the point $v$ belongs to $Z_{x} M$ if and only if there exist a real analytic path $\gamma:[0,1] \longrightarrow M, \gamma(0)=x$ satisfying $\frac{d \gamma}{d t}=v$. The same holds true for $Z_{x} M_{\mathbb{R}}$. Thus, $v \in Z_{x} M$ if and only if $v \in Z_{x} M_{\mathbb{R}}$.

\section{Singular hyperkähler varieties.}

In this section, we follow [V3], Section 10. For more examples, motivations and reference, the reader is advised to check [V3].

Definition 3.1. ([V-bun], Definition 6.5) Let $M$ be a hypercomplex variety (Definition 2.7). The following data define a structure of hyperkähler variety on $M$.

(i): For every $x \in M$, we have an $\mathbb{R}$-linear symmetric positively defined bilinear form $s_{x}: T_{x} M \times T_{x} M \longrightarrow \mathbb{R}$ on the corresponding real Zariski tangent space.

(ii): For each triple of induced complex structures $I, J, K$, such that $I \circ J=$ $K$, we have a holomorphic differential 2-form $\Omega \in \Omega^{2}(M, I)$.

(iii): Fix a triple of induced complex structure $I, J, K$, such that $I \circ J=$ $K$. Consider the corresponding differential 2 -form $\Omega$ of (ii). Let $J$ : $T_{x} M \longrightarrow T_{x} M$ be an endomorphism of the real Zariski tangent spaces defined by $J$, and $\left.R e \Omega\right|_{x}$ the real part of $\Omega$, considered as a bilinear form on $T_{x} M$. Let $r_{x}$ be a bilinear form $r_{x}: T_{x} M \times T_{x} M \longrightarrow \mathbb{R}$ defined by $r_{x}(a, b)=-\left.\operatorname{Re} \Omega\right|_{x}(a, J(b))$. Then $r_{x}$ is equal to the form $s_{x}$ of (i). In particular, $r_{x}$ is independent from the choice of $I, J, K$.

\section{Remark 3.2.}

(a): It is clear how to define a morphism of hyperkähler varieties.

(b): For $M$ non-singular, Definition 3.1 is equivalent to the usual one (Definition 1.1). If $M$ is non-singular, the form $s_{x}$ becomes the usual Riemann form, and $\Omega$ becomes the standard holomorphically symplectic form.

(c): It is easy to check the following. Let $X$ be a hypercomplex subvariety of a hyperkähler variety $M$. Then, restricting the forms $s_{x}$ and $\Omega$ to $X$, we obtain a hyperkähler structure on $X$. In particular, trianalytic subvarieties of hyperkähler manifolds are always hyperkähler, in the sense of Definition 3.1.

Caution. Not everything which looks hyperkähler satisfies the conditions of Definition 3.1. Take a quotient $M / G$ of a hyperkähler manifold by an action of finite group $G$, acting in accordance with hyperkähler structure. Then $M / G$ is, generally speaking, not hyperkähler (see [V3], Section 10 for details).

The following theorem, proven in [V-bun] (Theorem 6.3), gives a convenient way to construct examples of hyperkähler varieties.

Theorem 3.3. Let $M$ be a compact hyperkähler manifold, I an induced complex structure and $B$ a stable holomorphic bundle over $(M, I)$. Let $\operatorname{Def}(B)$ be 
a reduction ${ }^{1}$ of the deformation space of stable holomorphic structures on $B$. Assume that $c_{1}(B), c_{2}(B)$ are $S U(2)$-invariant, with respect to the standard action of $S U(2)$ on $H^{*}(M)$. Then $\operatorname{Def}(B)$ has a natural structure of a hyperkähler variety.

\section{Spaces with locally homogeneous singularities}

Definition 4.1. (local rings with LHS) Let $A$ be a local ring. Denote by $\mathfrak{m}$ its maximal ideal. Let $A_{g r}$ be the corresponding associated graded ring. Let $\hat{A}$, $\hat{A}_{g r}$ be the $\mathfrak{m}$-adic completion of $A, A_{g r}$. Let $(\hat{A})_{g r},\left(\hat{A}_{g r}\right)_{g r}$ be the associated graded rings, which are naturally isomorphic to $A_{g r}$. We say that $A$ has locally homogeneous singularities (LHS) if there exists an isomorphism $\rho: \hat{A} \longrightarrow \hat{A}_{g r}$ which induces the standard isomorphism $i:(\hat{A})_{g r} \longrightarrow\left(\hat{A}_{g r}\right)_{g r}$ on associated graded rings.

Definition 4.2. (SLHS) Let $X$ be a complex or real analytic space. Then $X$ is called be a space with locally homogeneous singularities (SLHS) if for each $x \in M$, the local $\operatorname{ring} \mathcal{O}_{x} M$ has locally homogeneous singularities.

By system of coordinates on a complex space $X$, defined in a neighbourhood $U$ of $x \in X$, we understand a closed embedding $U \hookrightarrow B$ where $B$ is an open subset of $\mathbb{C}^{n}$. Clearly, a system of coordinates can be considered as a set of functions $f_{1}, \ldots, f_{n}$ on $U$. Then $U \subset B$ is defined by a system of equations on $f_{1}, \ldots f_{n}$.

Remark 4.3. Let $X$ be a complex space. Assume that for each $x \in X$, there exist a system of coordinates $f_{1}, \ldots, f_{n}$ in a neighbourhood $U$ of $x$, such that $U \subset B$ is defined by a system of homogeneous polynomial equations. Then $X$ is a space with locally homogeneous singularities. This explains the term.

Claim 4.4. Let $X$ be a complex or real analytic space with locally homogeneous singularities, and $X_{r}$ its reduction Then $X_{r}$ is also a space with locally homogeneous singularities.

Proof. Clear.

Lemma 4.5. Let $A_{1}, A_{2}$ be local rings over $\mathbb{C}$, with $A_{i} / \mathfrak{m}_{i}=\mathbb{C}$, where $\mathfrak{m}_{i}$ is the maximal ideal of $A_{i}$. Then $A_{1} \otimes_{\mathbb{C}} A_{2}$ is LHS if and only if $A_{1}$ and $A_{2}$ are LHS.

Proof ("if" part). Let $\rho_{i}: \hat{A}_{i} \longrightarrow \widehat{\left(A_{i}\right)_{g r}}$ be the maps given by LHS condition. Consider the map

$$
\rho_{1} \otimes \rho_{2}: \hat{A}_{i} \otimes_{\mathbb{C}} \hat{A}_{2} \longrightarrow \widehat{\left(A_{i}\right)_{g r}} \otimes_{\mathbb{C}} \widehat{\left(A_{2}\right)_{g r}} .
$$

Denote the functor of adic completions of local rings by $B \longrightarrow \widehat{B}$. Clearly, $\widehat{A_{i}} \widehat{\otimes_{\mathbb{C}}} \hat{A}_{2}=\widehat{A_{1} \widehat{\otimes_{\mathbb{C}}} A_{2}}$, and $\left(\hat{A}_{i}\right)_{g r} \widehat{\otimes_{\mathbb{C}}}\left(\hat{A}_{2}\right)_{g r}=\left(A_{1}\right)_{g r} \widehat{\otimes_{\mathbb{C}}}\left(A_{2}\right)_{g r}$. Plugging

\footnotetext{
${ }^{1}$ The deformation space might have nilpotents in the structure sheaf. We take its reduction to avoid this.
} 
these isomorphisms into the completion of both sides of (4.1), we obtain that a completion of $\rho_{1} \otimes \rho_{2}$ provides an LHS map for $A_{1} \otimes_{\mathbb{C}} A_{2}$.

( "only if" part). Let

$$
\rho: \widehat{A_{1} \widehat{\otimes_{\mathbb{C}}} A_{2}} \longrightarrow\left(\left(A_{1}\right) \widehat{\otimes_{\mathbb{C}}}\left(A_{2}\right)\right)_{g r}
$$

be the LHS map for $A_{1} \otimes_{\mathbb{C}} A_{2}$. There are natural maps

$$
u: \hat{A}_{1} \longrightarrow \widehat{A_{1} \widehat{\otimes_{\mathbb{C}}} A_{2}}
$$

and

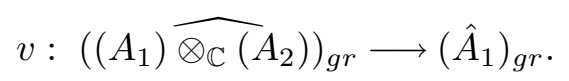

The $u$ comes from the natural embedding $a \longrightarrow a \otimes 1 \in A_{1} \otimes_{\mathbb{C}} A_{2}$ and $v$ from the natural surjection $a \otimes b \longrightarrow a \otimes \pi(b) \in A_{1} \otimes_{\mathbb{C}} \mathbb{C}$, where $\pi: A_{2} \longrightarrow \mathbb{C}$ is the standard quotient map. It is clear that $u \circ v$ induces identity on the associated graded ring of $A_{1}$. Lemma 4.5 is proven.

Proposition 4.6. Let $M$ be a complex variety, $M_{\mathbb{R}}$ the underlying real analytic space. Then $M_{\mathbb{R}}$ is a space with locally homogeneous singularities (SLHS) if and only if $M$ is SLHS.

Proof. By Claim 2.1, $\left(\mathcal{O}_{x} \widehat{M_{\mathbb{R}}}\right) \otimes \mathbb{C}=\mathcal{O}_{x} \widehat{M \otimes \overline{\mathcal{O}}_{x}} M$. Thus, Proposition 4.6 is implied immediately by Lemma 4.5 .

Corollary 4.7. Let $M$ be a hyperkähler (or hypercomplex) variety, $I_{1}, I_{2}$ induced complex structures. Then $\left(M, I_{1}\right)$ is a space with locally homogeneous singularities if and only is $\left(M, I_{2}\right)$ is $S L H S$.

Proof. The real analytic space underlying $\left(M, I_{1}\right)$ coinsides with that underlying $\left(M, I_{2}\right)$. Applying Proposition 4.6, we immediately obtain Corollary 4.7.

Definition 4.8. Let $M$ be a hyperkähler variety. Then $M$ is called a space with locally homogeneous singularities (SLHS) if the underlying real analytic space is SLHS or, equivalently, for some induced complex structure $I$ the $(M, I)$ is SLHS.

Theorem 4.9. Let $M$ be a compact hyperkähler manifold, I an induced complex structure and $B$ a stable holomorphic bundle over $(M, I)$. Assume that $c_{1}(B)$, $c_{2}(B)$ are $S U(2)$-invariant, with respect to the standard action of $S U(2)$ on $H^{*}(M)$. Let $\operatorname{Def}(B)$ be a reduction of a deformation space of stable holomorphic structures on $B$, which is a hyperkähler variety by Theorem 3.3. Then $\operatorname{Def}(B)$ is a space with locally homogeneous singularities (SLHS).

Proof. Let $x$ be a point of $\operatorname{Def}(B)$, corresponding to a stable holomorphic bundle $B$. In [V-bun], Section 7, the neighbourhood $U$ of $x$ in $\operatorname{Def}(B)$ was described explicitly as follows. We constructed a locally closed holomorphic embedding $U \stackrel{\varphi}{\hookrightarrow} H^{1}(\operatorname{End}(B))$. We proved that $v \in H^{1}(\operatorname{End}(B))$ belongs to the image of $\varphi$ if and only if $v^{2}=0$. Here $v^{2} \in H^{2}(\operatorname{End}(B))$ is the square of $v$, taken with respect to the product

$$
H^{1}(\operatorname{End}(B)) \times H^{1}(\operatorname{End}(B)) \longrightarrow H^{2}(\operatorname{End}(B))
$$


associated with the algebraic structure on $\operatorname{End}(B)$. Clearly, the relation $v^{2}=0$ is homogeneous. This relation defines a locally closed SLHS subspace $Y$ of $H^{1}(\operatorname{End}(B))$, such that $\varphi(U)$ is its reduction. Applying Claim 4.4, we obtain that $\varphi(U)$ is also a space with locally homogeneous singularities.

Theorem 4.10. Let $M$ be a hyperkähler variety. Then $M$ is a space with locally homogeneous singularities.

Proof. The paper [V-ne], which is a second part of the present paper, is fully taken by the proof of Theorem 4.10.

We don't use Theorem 4.10 in the present paper.

\section{Tangent cone of a hyperkähler variety}

Let $M$ be a hyperkähler variety, $I$ an induced complex structure and $Z_{x}(M, I)$ be a reduction of a Zariski tangent cone to $(M, I)$ in $x \in M$. Consider $Z_{x}(M, I)$ as a closed subvariety in the Zariski tangent space $T_{x} M$. The space $T_{x} M$ has a natural metric and quaternionic structure. This makes $T_{x} M$ into a hyperkähler manifold, isomorphic to $\mathbb{H}^{n}$.

Theorem 5.1. Under these assumptions, the following assertions hold:

(i): The subvariety $Z_{x}(M, I) \subset T_{x} M$ is independent from the choice of induced complex structure $I$.

(ii): Moreover, $Z_{x}(M, I)$ is a trianalytic subvariety of $T_{x} M$.

Proof. Theorem 5.1 (i) is implied by Proposition 2.9. By Theorem 5.1 (i), the Zariski tangent cone $Z_{x}(M, I)$ is a hypercomplex subvariety of $T M$. According to Remark $3.2(\mathrm{c})$, this implies that $Z_{x}(M)$ is hyperkähler.

Further on, we denote the Zariski tangent cone to a hyperkähler variety by $Z_{x} M$. The Zariski tangent cone is equipped with a natural hyperkähler structure.

The following theorem shows that the Zariski tangent cone $Z_{x} M \subset T_{x} M$ is a union of planes $L_{i} \subset T_{x} M$.

Theorem 5.2. Let $M$ be a hyperkähler variety, $I$ an induced complex structure and $x \in M$ a point. Consider the reduction of the Zariski tangent cone (denoted by $\left.Z_{x} M\right)$ as a subvariety of the quaternionic space $T_{x} M$. Let $Z_{x}(M, I)=\cup L_{i}$ be the irreducible decomposition of the complex variety $Z_{x}(M, I)$. Then

(i): The decomposition $Z_{x}(M, I)=\cup L_{i}$ is independent from the choice of induced complex structure $I$.

(ii): For every $i$, the variety $L_{i}$ is a linear subspace of $T_{x} M$, invariant under quaternion action.

Proof. Let $L_{i}$ be an irreducible component of $Z_{x}(M, I), Z_{x}^{n s}(M, I)$ be the nonsingular part of $Z_{x}(M, I)$, and $L_{i}^{n s}:=Z_{x}^{n s}(M, I) \cap L_{i}$. Then $L_{i}$ is a closure of $L_{i}^{n s}$ in $T_{x} M$. Clearly from Theorem $5.1, L_{i}^{n s}(M)$ is a hyperkähler submanifold in $T_{x} M$. By Proposition 1.8, $L_{i}^{n s}$ is totally geodesic. A totally geodesic submanifold 
of a flat manifold is again flat. Therefore, $L_{i}^{n s}$ is an open subset of a linear

subspace $\widetilde{L}_{i} \subset T_{x} M$. Since $L_{i}^{n s}$ is a hyperkähler submanifold, $\widetilde{L}_{i}$ is invariant with respect to quaternions. The closure $L_{i}$ of $L_{i}^{n s}$ is a complex analytic subvariety of $T_{x}(M, I)$. Therefore, $\widetilde{L}_{i}=L_{i}$. This proves Theorem 5.2 (ii). From the above argument, it is clear that $Z_{x}^{n s}(M, I)=\coprod L_{i}^{n s}$ (disconnected sum). Taking connected components of $Z_{x}^{n s} M$ for each induced complex structure, we obtain the same decomposition $Z_{x}(M, I)=\cup L_{i}$, with $L_{i}$ being closured of connected components. This proves Theorem 5.2 (ii).

Corollary 5.3. Let $M$ be a hyperkähler (or hypercomplex) variety, and $I$ an induced complex structure. Assume that $M$ is a space with locally homogeneous singularities. Then the normalization of $(M, I)$ is smooth.

Proof. The normalization of $Z_{x} M$ is smooth by Theorem 5.2. The normalization is compatible with the adic completions ([M], Chapter 9, Proposition 24.E). Therefore, the integral closure of the completion of $\mathcal{O}_{Z_{x} M}$ is a regular ring. Now, from the definition of locally homogeneous intersections, it follows that the integral closure of $\mathcal{O}_{x} M^{\sim}$ is also a regular ring, where $\mathcal{O}_{x} M^{\longleftarrow}$ is an adic completion of the local ring of holomorphic functions on $(M, I)$ in a neighbourhood of $x$. Applying [M], Chapter 9, Proposition 24.E again, we obtain that the integral closure of $\mathcal{O}_{x} M$ is regular. This proves Corollary 5.3

\section{Desingularization of hyperkähler varieties}

Theorem 6.1. Let $M$ be a hyperkähler or a hypercomplex variety. Assume that $M$ is a space with locally homogeneous singularities, and $I$ an induced complex structure. Let

$$
\widetilde{(M, I)} \stackrel{n}{\longrightarrow}(M, I)
$$

be the normalization of $(M, I)$. Then $\widetilde{(M, I)}$ is smooth and has a natural hyperkähler (respectively, hypercomplex) structure $\mathcal{H}$, such that the associated map $n: \widetilde{(M, I)} \longrightarrow(M, I)$ agrees with $\mathcal{H}$. Moreover, the hyperkähler manifold $\widetilde{M}:=\widetilde{(M, I)}$ is independent from the choice of induced complex structure $I$.

Proof. The variety $\widetilde{(M, I)}$ is smooth by Corollary 5.3. Let $x \in M$, and $U \subset M$ be a neighbourhood of $x$. Let $\Re_{x}(U)$ be the set of irreducible components of $U$


$\operatorname{Irr}\left(\operatorname{Spec} \mathcal{O}_{x} M^{\llcorner}\right)$is a set of irreducible components of $\operatorname{Spec} \mathcal{O}_{x} M^{\llcorner}$, where $\mathcal{O}_{x} M^{\curlyvee}$ is a completion of $\mathcal{O}_{x} M$ in $x$. Since $\mathcal{O}_{x} M$ is Henselian ([R], VII.4), there exist

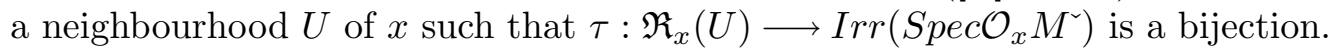
Fix such an $U$. Since $M$ is a space locally with locally homogeneous singularities, the irreducible decomposition of $U$ coinsides with the irreducible decomposition of the tangent cone $Z_{x} M$.

Let $\amalg U_{i} \stackrel{u}{\longrightarrow} U$ be the morphism mapping a disjoint union of irreducible components of $U$ to $U$. By Theorem 5.2, the $x$-completion of $\mathcal{O}_{U_{i}}$ is regular. 
Shrinking $U_{i}$ if necessary, we may assume that $U_{i}$ is smooth. Then, the morphism $u$ coinsides with the normalization of $U$.

For each variety $X$, we denote by $X^{n s} \subset X$ the set of non-singular points of $X$. Clearly, $u\left(U_{i}\right) \cap U^{n s}$ is a connected component of $U^{n s}$. Therefore, $u\left(U_{i}\right)$ is trianalytic in $U$. By Remark 3.2 (c), $U_{i}$ has a natural hyperkähler structure, which agrees with the map $u$. This gives a hyperkähler structure on the normalization $\widetilde{U}:=\amalg U_{i}$. Gluing these hyperkähler structures, we obtain a hyperkähler structure $\mathcal{H}$ on the smooth manifold $\widetilde{(M, I)}$. Consider the normalization map $n: \widetilde{(M, I)} \longrightarrow M$, and let $\widetilde{M}^{n}:=n^{-1}\left(M^{n s}\right)$. Then, $\left.n\right|_{\widetilde{M}^{n}} \widetilde{M}^{n} \longrightarrow M^{n s}$ is a finite covering which is compatible with the hyperkähler structure. Thus, $\left.\mathcal{H}\right|_{\widetilde{M}^{n}}$ can be obtained as a pullback from $M$. Clearly, a hyperkähler structure on a manifold is uniquely defined by its restriction to an open dense subset. We obtain that $\mathcal{H}$ is independent from the choice of $I$.

Remark 6.2. The desingularization argument works well for hypercomplex varieties. The word "hyperkähler" in this article can be in most cases replaced by "hypercomplex", because we never use the metric structure.

\section{Acknowledgements}

It is a pleasure to acknowledge the help of P. Deligne, who pointed out an error in the original argument, and another error in the presentation. Deligne also suggested the term "locally homogeneous singularities". I am grateful to A. Beilinson, D. Kaledin, D. Kazhdan, T. Pantev and S.-T. Yau for enlightening discussions.

\section{References}

[Beau] A. Beauville, Variétés Kähleriennes dont la première classe de Chern est nulle, J. Diff. Geom. 18 (1983), 755-782.

[Bes] A. Besse, Einstein manifolds, Springer-Verlag, New York (1987).

[C] E. Calabi, Metriques kähleriennes et fibrès holomorphes, Ann. Ecol. Norm. Sup. 12 (1979), 269-294.

[GMT] F. Guaraldo,P. Macri, A. Tancredi, Topics on real analytic spaces, Adv. Lectures Math., Braunschweig: F. Vieweg, 1986.

[H] A. Hartshorne, Algebraic geometry, Grad. Texts in Math., vol. 52, Springer-Verlag, New York, 1977.

[M] H. Matsumura, Commutative algebra, Math. Lecture Note Ser., vol. 56, Benjamin/Cummings Pub. Co., Reading, Mass., 1980.

[R] M. Raynaud, Anneaux locaux Henséliens, Springer, Lecture Notes in Math. 169, 1970. 
[V-bun] M. Verbitsky, Hyperholomorphic bundles over a hyperkähler manifold, alg-geom electronic preprint 9307008 (1993), 43 pages, also published in: J. Alg. Geom. 5 (1996), $633-669$.

[V2] _ Hyperkähler embeddings and holomorphic symplectic geometry II, alg-geom electronic preprint 9403006 (1994), 14 pages, also published in: Geom. Funct. Anal. 5 (1995), 92-104.

[V3] _ Deformations of trianalytic subvarieties of hyperkähler manifolds, alg-geom electronic preprint 9610010 (1996), 51 pages.

[V-ne] , Desingularization of singular hyperkähler varieties II, alg-geom/9612013, 15 pages.

Institute for Advanced Study, Princeton, NJ 08540

E-mail address: verbit@thelema.dnttm.rssi.ru, verbit@math.ias.edu 